\title{
Displacement of the pisiform: a radiographic sign for synovitis of the wrist joint
}

\author{
Matthew Morris • Peter M. Murray • Mark Kransdorf
}

Received: 13 January 2011 /Revised: 22 June 2011 /Accepted: 4 July 2011 / Published online: 13 August 2011

(C) ISS 2011

\begin{abstract}
Answer
Acute traumatic wrist synovitis causing volar displacement of the pisiform.

The patient presented to the emergency department and was diagnosed with a dislocation of the pisiform, and her wrist was splinted. Upon examination in the hand clinic, ulnar-sided wrist pain and warmth were noted. MRI examination demonstrated an enhancing proliferation of tissue and fluid on $\mathrm{T} 2$, which emanated from the radiocarpal joint, displacing the pisiform volarly. The flexor carpi ulnaris tendon insertion was deemed intact.

Isolated dislocations of the pisiform are rare. Traumatic synovitis of the wrist joint results from inflammatory proliferation of the synovium resulting in symptoms of pain, swelling, and restricted motion [1]. The most common noninfectious cause of traumatic wrist synovitis is overuse or trauma [2]. The 1 week delay between injury and onset of symptoms in this case is more characteristic of traumatic wrist synovitis. Traumatic wrist synovitis can also be seen in patients with underlying conditions such as rheumatoid arthritis, osteoarthritis, crystalline deposition disease (CPPD), or infection [2]. No evidence of rheumatoid arthritis or CPPD was seen on radiographs. The patient was placed in a right wrist splint and given a 6 day Medrol Dosepak. Follow
\end{abstract}

up examination at 2 weeks showed complete resolution of wrist swelling, tenderness, and warmth.

The pisiform triquetral joint is a synovial joint that communicates with the radiocarpal joint [3]. In this case the traumatic synovitis proliferation from the wrist joint expanded into the pisiform triquetral joint causing the displacement of the pisiform. This was demonstrated on MR by the acute angle subtended by the FCU tendon. The MRI findings as well as the clinical features of ulnar-sided wrist pain, wrist swelling, and the prompt resolution of symptoms lead to the diagnosis of acute traumatic wrist synovitis. We now consider displacement of the pisiform on conventional wrist radiographs a potential sign of acute wrist synovitis.

\section{References}

1. Boles DS, Schmidt C. Pyogenic flexor tenosynovitis. Hand Clin. 1998;14(4):567-77.

2. Bullocks JM, Downey C, Gonzalez Gibler P, Netscher D. Crystal deposition disease masquerading as proliferative tenosynovitis and its associated sequelae. Ann Plast Surg. 2009;62:128-33.

3. Theumann NH, Pfirrmann CW, Chung CB, Antonio GE, Trudell DJ, Resnick D. Pisotriquetral joint: assessment with MR imaging and MR arthrography. Radiology. 2002;222:763-77.

The case presentation can be found at doi:10.1007/s00256-011-1235-y.

M. Morris · P. M. Murray $(\bowtie) \cdot$ M. Kransdorf

Department of Orthopedics, Mayo Clinic,

4500 San Pablo Road South,

Jacksonville, FL 32224, USA

e-mail: murray.peter@mayo.edu 